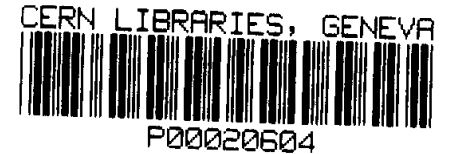

CERN-TH.7101/93

THEF-NYM 93.16

\title{
Deformed Poincaré containing the exact Lorentz algebra*
}

\author{
Alexandros A. Kehagias ${ }^{\dagger}$, Patrick A. A. Meessen $\ddagger$ \\ Institute of Theoretical Physics \\ University of Nijmegen, Toernooiveld 1, \\ 6525 ED, Nijmegen, The Netherlands \\ and \\ George Zoupanos $\S$ \\ Theory Division, CERN \\ CH-1211 Geneva 23, Switzerland
}

\begin{abstract}
A deformed Poincare algebra that contains the exact Lorentz algebra is constructed. By imposing further constraints, the way is paved towards the construction of field theories with improved renormalization properties. The space-time structure and the representations of the constructed deformed Poincaré algebra are also discussed.
\end{abstract}

CERN-TH.7101/93

December 1993

*Partially supported by CEC Science project No. SC1-CT91-0729

${ }^{\dagger}$ Partially supported by CEC Contract No. ERBCHBGCT 920197

$\ddagger$ Partially supported by 'de Wet op Studiefinanciering' No. 5614-71105-0-13

${ }^{\S}$ Permanent address: Phys. Dept., National Technical University, 15780 Zografou, Athens, Greece 


\section{Introduction}

In the recent years quantum deformations of space-time symmetries have attracted an appreciable amount of interest [1-9]. Among other reasons explaining this intensive research activity is the fact that q-deformations of space-time seem to lead to some lattice pattern. Defining quantum field theories on a lattice is a well-known regularization procedure and has been used very extensively. One of the negative aspects of the most popular way to do that, which uses equidistance lattices, is that it spoils the Lorentz invariance of the theory ${ }^{1}$. By deforming the symmetries of space-time one can hope for improvement of the ultraviolet properties of a quantum field theory defined on it. More specifically, it is fair to hope that the space-time symmetries, although deformed, are still remembered by the theory, while in the worst case some kind of regularization is built in. Next it is natural to search for deformations of space-time symmetries that maintain as much as possible of those required by the known renormalizable field theories describing elementary particle physics. In this spirit we examine here deformations of the Poincare algebra (PA), which do not affect the Lorentz algebra. Among other things, this will permit us to define field theories for particles with usual spin, which in turn is expected to facilitate their quantization.

There exists a well-established formalism to obtain the q-deformed counterpart of a simple Lie algebra developed by Drinfeld and Jimbo [11]. However, the PA is not simple since it is the semi-direct sum of the Lorentz and the translation subalgebras: $\mathcal{P} \simeq O(3,1) \boxplus T_{4}$. As a result another scheme must be found in order to construct a deformed Poincaré algebra (dPA).

There exist, mainly, two possible constructions. The direct one involves the employment of the q-deformed Lorentz algebra, which is isomorphic to $S L_{q}(2, C)$ and associates to it a q-deformed four-vector, which is interpreted as the four-momentum [3]. This construction obviously is not aligned with the strategy we would like to develop here.

The other construction is based on the fact that the PA can be obtained by a WignerInönü contraction of the simple anti-de Sitter algebra $O(3,2)[4,5]$. Thus, one first constructs the quantum group $O_{q}(3,2)$ using the Drinfeld-Jimbo method and after the introduction of the anti-de Sitter radius $R$, the contraction

$$
R \rightarrow \infty \quad, \quad i R \log q \rightarrow \kappa^{-1}
$$

is performed. A very interesting feature of this construction is the introduction of a dimensionful parameter $\kappa$ and the ordinary PA is recovered in the limit $\kappa \rightarrow \infty$. The same result has been obtained by Bacry [8] who directly considered general deformations for the sets of generators $\left(K_{i}, K_{j}\right),\left(K_{i}, P_{0}\right)$ and $\left(K_{i}, P_{j}\right)$. Unfortunately, this dPA does not contain the Lorentz algebra either.

In addition, the light-cone depends on the energy of the photons. As a result the lightcone is not well defined since its angle is not uniquely specified and depends on the energy [8]. This creates problems with causality and the natural way to avoid such problems is again to demand the existence of the exact Lorentz subalgebra in the dPA. In that case causality is guaranteed as in the ordinary case.

\footnotetext{
${ }^{1}$ There exist Lorentz-invariant formulations of field theories on random lattices [10].
} 


\section{Deformed Poincaré containing the exact Lorentz algebra}

Let us recall some well-known properties of the PA. This is a ten-dimensional Lie algebra whose generators can be labelled $J_{i}, K_{i}, P_{i}$ and $P_{0}(i=1,2,3)$. It is defined by the following commutation relations

$$
\begin{aligned}
{\left[J_{i}, J_{j}\right] } & =i \epsilon_{i j k} J_{k}, \\
{\left[J_{i}, K_{j}\right] } & =i \epsilon_{i j k} K_{k}, \\
{\left[J_{i}, P_{j}\right] } & =i \epsilon_{i j k} P_{k}, \\
{\left[J_{i}, P_{0}\right] } & =0 \\
{\left[P_{i}, P_{j}\right] } & =0 \\
{\left[P_{i}, P_{0}\right] } & =0 \\
{\left[K_{i}, P_{0}\right] } & =i P_{i} \\
{\left[K_{i}, P_{j}\right] } & =i P_{0} \delta_{i j}, \\
{\left[K_{i}, K_{j}\right] } & =-i \epsilon_{i j k} J_{k} .
\end{aligned}
$$

As may been seen from eqs.(2)-(10), $J_{i}, K_{i}$ and $P_{i}$ are three-vectors generating rotations, boosts and translations, respectively; $P_{0}$ corresponds to the energy and, according to eq.(5), is a scalar. The PA contains the Lorentz algebra as a subalgebra, which is generated by $J_{i}$ 's and $K_{i}$ 's and the commutation relations $(2),(3),(10)$.

The PA has two quadratic Casimir invariants. They are the lengths of the momentum four-vector $P_{\mu}=\left(P_{0}, P_{i}\right)$ :

$$
P_{\mu} P^{\mu}=P_{0}^{2}-\vec{P} \cdot \vec{P},
$$

and the Pauli-Lubanski four-vector $W_{\mu}=\left(J_{i} P_{i}, P_{0} J_{i}+\epsilon_{i j k} P_{j} K_{k}\right)$ :

$$
W_{\mu} W^{\mu}=W_{0}^{2}-\vec{W} \cdot \vec{W} .
$$

These invariants label the representations of the PA.

The enlargement of the Lorentz group to the Poincaré group was proposed [13] as a way of describing the quantum states of relativistic particles without using the wave equations. The states of a free particle are then given by the unitary irreducible representations of the Poincare group. One of our main aims in the following is to show that the above enlargement of the Lorentz algebra is not unique. The solution that we propose can be seen as a deformation of the Poincaré algebra. Clearly this deformation will be defined in the enveloping algebra of the PA. The latter is generated by all possible polynomials of the generators, and the deformed algebra we are looking for will form a closed subset of this enveloping algebra. There are many ways to choose this subset, of which we have already mentioned two in the Introduction. Moreover we demand that the dPA thus constructed have a Casimir invariant of the form

$$
f\left(P_{0}\right)-\vec{P} \cdot \vec{P}
$$

which will correspond to the (mass) ${ }^{2}$ in the ordinary case. 
A very important feature of the Lorentz-invariant Casimir (13), which is a deformation of the Casimir (11), is that it only changes the $P_{0}$-part of the latter. At first sight one might think that it is impossible to define a Lorentz-invariant Casimir without deforming also its $\vec{P}$-part. In fact the authors in ref.[5, 8], who also demanded the existence of a Casimir invariant of the form (13), were led considering a deformation of the Lorentz algebra as well. Therefore one might find it quite striking that such a deformation can be done in a Lorentz-invariant way.

Let us discuss explicitly how the above features can be realized. Keeping the Lorentz algebra unchanged we enlarge the set of generators $\left(J_{i}, K_{j}\right)$ with a 3 -vector $P_{i}$ and a scalar $P_{0}$. In this way the commutation relations $(4),(5)$ remain unchanged as compared to the ordinary PA. Next we choose, for the purposes of the present paper, to keep also the commutation relations among the generators $\left(P_{0}, P_{i}\right)(6),(7)$ as in the ordinary PA. The reason for the latter choice is that we are interested here in introducing ultraviolet regularizations. Introducing non-commutativity in the $P_{i}$ 's would provide an infrared regularization. Proceeding in this way we write down generalized commutation relations (as compared to the ordinary PA) for the generators $\left(P, K_{i}\right)$ :

$$
\begin{aligned}
& {\left[K_{i}, P_{0}\right]=i \alpha_{i}\left(P_{0}, \vec{P}\right),} \\
& {\left[K_{i}, P_{j}\right]=i \beta_{i j}\left(P_{0}, \vec{P}\right) .}
\end{aligned}
$$

Here $\alpha_{i}, \beta_{i j}$ are functions of $P_{0}$ and $P_{j}$.

The generators of the dPA have to satisfy the Jacobi identities. Applying the Jacobi identity to the set $\left(J_{i}, K_{i}, P_{0}\right)$, we conclude that $\alpha_{i}$ must be a vector, i.e.

$$
\left[J_{i}, \alpha_{j}\right]=i \epsilon_{i j k} \alpha_{k} .
$$

Correspondingly, the Jacobi identity for the set $\left(J_{i}, K_{j}, P_{k}\right)$ determines that $\beta_{i j}$ transforms as a symmetric tensor under $\mathrm{SU}(2)$ :

$$
\left[J_{i}, \beta_{j l}\right]=i \epsilon_{i j k} \beta_{k l}+i \epsilon_{i l k} \beta_{j k} .
$$

From eqs.(16),(17) it follows that $\alpha_{i}$ and $\beta_{j k}$ can be chosen as

$$
\begin{aligned}
\alpha_{i}\left(P_{0}, \vec{P}\right) & =\alpha\left(P_{0}, P^{2}\right) P_{i}, \\
\beta_{j k}\left(P_{0}, \vec{P}\right) & =\beta\left(P_{0}, P^{2}\right) \delta_{j k}+\gamma\left(P_{0}, P^{2}\right) P_{j} P_{k} .
\end{aligned}
$$

Furthermore, we have assumed the expression (13) is invariant under the dPA and thus commutes with all generators. One can easily check that $(13)$ indeed commutes with $J_{i}, P_{i}, P_{0}$. The commutator of $K_{i}$ with expression (13) gives

$$
\begin{aligned}
f^{\prime}\left(P_{0}\right) & =2 \frac{\beta\left(P_{0}, P^{2}\right)}{\alpha\left(P_{0}, P^{2}\right)}, \\
\gamma\left(P_{0}, P^{2}\right) & =0
\end{aligned}
$$

where $f^{\prime}$ denotes the derivative of $f$ with respect to $P_{0}$, and, consequently:

$$
\begin{aligned}
\alpha_{i}\left(P_{0}, P^{2}\right) & =\alpha\left(P_{0}\right) P_{i}, \\
\beta_{j k}\left(P_{0}, P^{2}\right) & =\beta\left(P_{0}\right) \delta_{j k} .
\end{aligned}
$$


The functions $\alpha$ and $\beta$ in eq.(20) are not independent. One may easily see that from the Jacobi identity for the set $\left(K_{i}, K_{j}, P_{j}\right)$, i.e.

$$
\left[K_{i},\left[K_{j}, P_{k}\right]\right]+\left[K_{j},\left[P_{k}, K_{i}\right]\right]+\left[P_{k},\left[K_{i}, K_{j}\right]\right]=0,
$$

we obtain, for $i=j$ :

$$
3\left(\beta^{\prime}\left(P_{0}\right) \alpha\left(P_{0}\right)-1\right) P_{k}=0 .
$$

As a result, in order to close the algebra, we must require that

$$
\alpha\left(P_{0}\right) \beta^{\prime}\left(P_{0}\right)=1
$$

In this way a minimally deformed Poincare algebra is defined by the commutation relations

$$
\begin{aligned}
{\left[J_{i}, J_{j}\right] } & =i \epsilon_{i j k} J_{k}, \\
{\left[J_{i}, K_{j}\right] } & =i \epsilon_{i j k} K_{k}, \\
{\left[J_{i}, P_{j}\right] } & =i \epsilon_{i j k} P_{k}, \\
{\left[K_{i}, K_{j}\right] } & =-i \epsilon_{i j k} J_{k}, \\
{\left[J_{i}, P_{0}\right] } & =0, \\
{\left[P_{i}, P_{j}\right] } & =\left[P_{i}, P_{0}\right]=0, \\
{\left[K_{i}, P_{0}\right] } & =i \alpha\left(P_{0}\right) P_{i}, \\
{\left[K_{i}, P_{j}\right] } & =i \beta\left(P_{0}\right) \delta_{i j},
\end{aligned}
$$

where $\alpha\left(P_{0}\right), \beta\left(P_{0}\right)$ satisfy eq.(23).

It should be noted that more general deformations can be performed as well [8]. However, the algebra (23)-(31) has the characteristic feature that it contains the exact Lorentz algebra.

Let us turn now to the Casimir invariants of the algebra (24)-(31). There exist two 'quadratic' Casimir invariants. One corresponds to the (mass) ${ }^{2}$ of the ordinary Poincaré algebra, and in view of eqs.(13),(19),(23) is given by

$$
\beta^{2}\left(P_{0}\right)-\vec{P} \cdot \vec{P}=\mu^{2} \text {. }
$$

The other corresponds to the length of the Pauli-Lubanski four-vector

$$
\begin{aligned}
& W_{0}=\vec{J} \cdot \vec{P} \\
& W_{i}=\beta\left(P_{0}\right) J_{i}+\epsilon_{i j k} P_{j} K_{k} .
\end{aligned}
$$

One can easily verify that the vector $(33),(34)$ satisfies the following commutation relations:

$$
\begin{aligned}
{\left[W_{0}, W_{i}\right] } & =i \epsilon_{i j k} \beta\left(P_{0}\right) J_{j} P_{k}+i K_{i}(\vec{P} \cdot \vec{P})-i P_{i}(\vec{K} \cdot \vec{P})+\beta\left(P_{0}\right) P_{i} \\
{\left[W_{i}, W_{j}\right] } & =i \epsilon_{i j k}\left(\beta^{2}\left(P_{0}\right) J_{k}-W_{0} P_{k}\right)+i \beta\left(P_{0}\right)\left(P_{i} K_{j}-P_{j} K_{i}\right), \\
{\left[J_{i}, W_{j}\right] } & =i \epsilon_{i j k} W_{k}, \\
{\left[J_{i}, W_{0}\right] } & =0 \\
{\left[K_{i}, W_{0}\right] } & =i W_{i}, \\
{\left[K_{i}, W_{j}\right] } & =i W_{0} \delta_{i j}, \\
{\left[P_{i}, W_{0}\right] } & =\left[P_{i}, W_{j}\right]=0 \\
{\left[P_{0}, W_{0}\right] } & =\left[P_{0}, W_{j}\right]=0 .
\end{aligned}
$$


Consequently, all generators commute with

$$
\mathcal{W}^{2}=W_{0}^{2}-\vec{W} \cdot \vec{W}
$$

i.e. the second Casimir invariant of the deformed algebra. The eigenvalues of $\mathcal{W}^{2}$ are

$$
\mathcal{W}^{2}=-\mu^{2} s(s+1)
$$

where $s=0, \frac{1}{2}, 1, \ldots$ is the spin, and will label the representations of the dPA.

Notice that the form of the $\beta$ function is not specified by the dPA. Therefore one can impose some physical requirements on the form of the $\beta$. An obvious one is to recover the ordinary PA in the low-energy region. This means that the low-energy behaviour of $\beta$ has to be

$$
\beta\left(P_{0}\right) \sim P_{0}
$$

Another constraint will be introduced by demanding that there exist an upper cut-off in the mass spectrum and, consequently, due to eq.(32), to the momentum for $\mu^{2}$ positive. This constraint means that the equation

$$
\beta^{\prime}\left(P_{0}\right)=0
$$

has a solution for a finite $P_{0}$. An obvious realization of such a function is provided by

$$
\beta\left(P_{0}\right)=M \sin \left(\frac{P_{0}}{M}\right),
$$

which, modulo periodicity, may restrict the energy $P_{0}$ in the interval

$$
-\frac{\pi M}{2} \leq P_{0} \leq \frac{\pi M}{2}
$$

Note that $\beta$ is an odd function and, thus, the representations of the dPA can be classified according to

$$
\operatorname{sign}(\beta)=\frac{\beta\left(P_{0}\right)}{\left|\beta\left(P_{0}\right)\right|} .
$$

Let us make further remarks concerning eq.(23). This equation holds independently of the representations. In the case that eq.(39) holds, we may define states denoted by $|M\rangle$ which are annihilated by $\beta^{\prime}$. Observe that $|M\rangle$ can be defined as a limiting case, i.e.,

$$
\lim _{\beta \rightarrow M}|\mu\rangle=|M\rangle \text {. }
$$

In that case we see that eq.(21) is satisfied only if the commutator $\left[K_{i}, K_{j}\right]$ also vanishes and thus leaves the $\alpha\left(P_{0}\right)$ unconstrained. The commutator $\left[K_{i}, \mu^{2}\right]$ acting on $|M\rangle$ gives zero only if $P_{i}$ also annihilates $|M\rangle$. As a result, the dPA acting on these states is realized by the algebra generated by the set $\left(J_{i}, K_{i}, P_{i}\right)$, which satisfies the commutation relations $(24)-(26),(29)$ and

$$
\begin{aligned}
{\left[K_{i}, K_{j}\right] } & =0 \\
{\left[K_{i}, P_{j}\right] } & =i \beta_{0} \delta_{i j} \\
{\left[K_{i}, P_{0}\right] } & =0
\end{aligned}
$$


where $\beta_{0}$ is the eigenvalue of $\beta\left(P_{0}\right)$ on $|M\rangle$. It is clear now that this algebra is no longer the dPA.

The states $|M\rangle$ which are annihilated by $\beta^{\prime}$, carry zero momentum and have maximum energy, which cannot be changed by the action of the boosts. A representation for the generators of the $\mathrm{dPA}$ in momentum space is given by

$$
\begin{aligned}
P_{0} & =p_{0} \\
P_{i} & =p_{i} \\
J_{i} & =-i \epsilon_{i j k} p_{j} \frac{\partial}{\partial p_{k}} \\
K_{i} & =i\left(p_{i} \alpha\left(p_{0}\right) \frac{\partial}{\partial p_{0}}+\beta\left(p_{0}\right) \frac{\partial}{\partial p_{i}}\right) .
\end{aligned}
$$

It is not difficult to verify that the above generators indeed satisfy the commutation relations (24) $-(31)$, if we take eq.(23) into account.

Let us finally make some remarks concerning the additivity properties of momentum $\vec{P}^{(12)}$ and energy $P_{0}^{(12)}$ of a system $S^{(12)}$ composed out of two non-interacting systems $S^{(1)}, S^{(2)}$ with momenta $\left.\vec{P}^{(1)}, \vec{P}^{(2)}\right)$ and energies $P_{0}^{(1)}, P_{0}^{(2)}$, respectively. Among the new ones, only the $\vec{P}^{(12)}, \vec{J}^{(12)}$ and $\vec{K}^{(12)}$ have the usual additivity property, i.e.:

$$
\begin{aligned}
\vec{P}^{(12)} & =\vec{P}^{(1)}+\vec{P}^{(2)} \\
\vec{J}^{(12)} & =\vec{J}^{(1)}+\vec{J}^{(2)} \\
\vec{K}^{(12)} & =\vec{K}^{(1)}+\vec{K}^{(2)}
\end{aligned}
$$

As far as the energy is concerned, although it is still conserved, the energy $P_{0}^{(12)}$ of $S^{(12)}$ is no longer the sum of the energies of the two subsystems $S^{(1)}$ and $S^{(2)}$. Instead we have

$$
\sin \frac{P_{0}^{(1)}}{M}+\sin \frac{P_{0}^{(2)}}{M}=2 \sin \frac{P_{0}^{(12)}}{2 M} \text {. }
$$

\section{Space-time of the deformed Poincaré algebra}

The dPA defined above has been constructed solely in momentum space. However, one can recover space-time, in the spirit of Gel'fand, as the spectrum of appropriate self-adjoint operators acting on momentum space. In order to do that we will assume that there exists a set of commuting operators $T, X_{i}(i=1,2,3)$ that satisfy

$$
\begin{aligned}
{\left[X_{i}, X_{j}\right] } & =\left[T, X_{i}\right]=0 \\
{\left[X_{i}, P_{j}\right] } & =i \delta_{i j}, \\
{\left[X_{i}, P_{0}\right] } & =0 \\
{\left[T, P_{0}\right] } & =i \alpha\left(P_{0}\right) .
\end{aligned}
$$

These operators act on functions on the momentum space and their eigenvalues $\left(t, x_{i}\right)$ label the space-time points. From eqs.(49) we see that a representation of $T, X_{i}$ is given by

$$
T=i \alpha\left(p_{0}\right) \frac{\partial}{\partial p_{0}}
$$




$$
X_{i}=i \frac{\partial}{\partial p_{i}}
$$

This particular representation satisfies the commutation relations

$$
\begin{aligned}
{\left[J_{i}, X_{j}\right] } & =i \epsilon_{i j k} X_{k} \\
{\left[J_{i}, T\right] } & =0 \\
{\left[K_{i}, X_{j}\right] } & =-i T \delta_{i j} \\
{\left[K_{i}, T\right] } & =-i X_{i} .
\end{aligned}
$$

It is now clear that the set $\left(T, X_{i}\right)$ transforms as a Lorentz four-vector. As a result Lorentz transformations leave invariant the quadratic expression

$$
T^{2}-\vec{X}^{2}
$$

Let us next find the domain $D\left(T, X_{i}\right)$ where these operators are self-adjoint. The function $\beta\left(p_{0}\right)$ has local extrema at the points $p_{0}= \pm \frac{\pi M}{2}$. We define the inner product for functions $f$ and $g$ as

$$
\langle f \mid g\rangle=\int d^{4} p \beta^{\prime}\left(p_{0}\right) f^{*}\left(p_{0}, \vec{p}\right) g\left(p_{0}, \vec{p}\right) .
$$

The operator $T$ is self-adjoint with respect to this inner product for the functions $f\left(p_{0}, \vec{p}\right)$ that satisfy

$$
f\left(\frac{\pi M}{2}, \vec{p}\right)=f\left(-\frac{\pi M}{2}, \vec{p}\right)=0 .
$$

The eigenvalues of the operator $T$ will be real and will correspond to the possible values of time measurements. These eigenvalues are specified by solving

$$
-i \frac{\partial}{\partial p_{0}} f\left(p_{0}, \vec{p}\right)=t \beta^{\prime}\left(p_{0}\right) f\left(p_{0}, \vec{p}\right)
$$

with the condition (54). The general solution of eq.(55) can be written as

$$
f\left(p_{0}, \vec{p}\right)=\sum_{n}\left[C_{n}(\vec{p}) \cos \left(\beta\left(p_{0}\right) \frac{(2 n+1) \pi}{2 M}\right)+D_{n}(\vec{p}) \sin \left(\beta\left(p_{0}\right) \frac{n \pi}{M}\right)\right] .
$$

where $M \equiv \beta\left(\frac{\pi M}{2}\right)$. The Fourier transform of the above solution in $t$-space is given by

$$
\begin{aligned}
f(t, \vec{x})= & \sum_{n}\left\{C_{n}^{\prime}(\vec{x})\left[\delta\left(t+\frac{(2 n+1) \pi}{2 M}\right)+\delta\left(t-\frac{(2 n+1) \pi}{2 M}\right)\right]\right. \\
& \left.+D_{n}^{\prime}(\vec{x})\left[\delta\left(t+\frac{n \pi}{M}\right)-\delta\left(t-\frac{n \pi}{M}\right)\right]\right\} .
\end{aligned}
$$

This leads to a lattice pattern for the $t$-axis with lattice-spacing $\frac{1}{M}$.

Let us now examine the $X_{i}$ spectrum. We recall that, in momentum space, three types of four-momenta exist: time-like $\left(\mu^{2}>0\right)$, space-like $\left(\mu^{2}<0\right)$ and null ones $\left(\mu^{2}=0\right)$. For non space-like momenta we see that $P^{2}$ is always less than or equal to $\beta^{2}$. If $\beta^{\prime}=0$ for some 
$p_{0}$, then there exists, as we have already discussed, a highest state with maximum energy $\frac{\pi M}{2} ; P^{2}$ is then always less than $M^{2}$, and thus

$$
-M \leq p \leq M
$$

In this respect, there also exist three types of $X_{i}$ : those that act on functions $f\left(p_{0}, \vec{p}\right)$, with $\left(p_{0}, \vec{p}\right)$ time-like, space-like or null four-momenta, respectively. For time-like momenta, proceeding as before, one can find that the coefficients $C_{n}$ in eq.(57) are given by

$$
\begin{aligned}
C^{\prime}{ }_{m}(\vec{x})= & \prod_{i=1}^{3} \sum_{n_{i}}\left\{E_{m, n_{i}}\left[\delta\left(x_{i}-\frac{\left(2 n_{i}+1\right) \pi}{2 M}\right)+\delta\left(x_{i}+\frac{\left(2 n_{i}+1\right) \pi}{2 M}\right)\right]\right. \\
& \left.+F_{m, n_{i}}\left[\delta\left(x_{i}+\frac{n_{i} \pi}{M}\right)-\delta\left(x_{i}-\frac{n_{i} \pi}{M}\right)\right]\right\} .
\end{aligned}
$$

and a similar expression for $D^{\prime}{ }_{m}(\vec{x})$. For space-like momenta these coefficients are continuous functions of $x_{i}$. As a result the space-time portrait looks like the following. At each spacetime point we can draw the curves given by equating expression (52) to zero. These curves specify the lightcone at that point and are invariant under Lorentz transformations. As in the ordinary case we define time-like, space-like and null regions. The fundamental difference is that for non-space-like regions a lattice structure emerges, whereas space-like ones are continuous [12].

Let us define the inner product of two vectors $|f\rangle,|g\rangle$ on the Hilbert space of a massive particle by

$$
\begin{aligned}
\langle f \mid g\rangle & =\int \frac{d^{4} p}{(2 \pi)^{4}} \delta\left(\beta^{2}\left(P_{0}\right)-p^{2}-\mu^{2}\right) \theta\left(p_{0}\right) f^{*}(p) g(p) \\
& =\int \frac{d^{3} p}{(2 \pi)^{3} 2 \beta^{\prime} \beta} f^{*}(\vec{p}) g(\vec{p})
\end{aligned}
$$

where $p_{0}$ is the positive solution of $\beta^{2}\left(p_{0}\right)-p^{2}=\mu^{2}$. A representation of the generators of the dPA is given by

$$
\begin{aligned}
P_{i} & =p_{i} \\
J_{i} & =-i \epsilon_{i j k} p_{j} \frac{\partial}{\partial p_{k}} \\
K_{i} & =i\left(\beta\left(p_{0}\right) \frac{\partial}{\partial p_{i}}+\frac{\alpha^{\prime}}{2} p_{i}\right) .
\end{aligned}
$$

These operators act on functions of the Hilbert space and they are self-adjoint with respect to the inner product $(60)$. Furthermore we can define a position operator by

$$
Q_{i} \equiv \frac{1}{2 \beta} K_{i}+K_{i} \frac{1}{2 \beta}
$$

which is Hermitean with respect to the inner product $(60)[8,14]$. It is easy to check that $Q_{i}$ is a vector, i.e.

$$
\left[J_{i}, Q_{j}\right]=i \epsilon_{i j k} Q_{k}
$$


and moreover

$$
\begin{aligned}
{\left[Q_{i}, Q_{j}\right] } & =0 \\
{\left[Q_{i}, P_{j}\right] } & =i \delta_{i j} .
\end{aligned}
$$

Using the representation (60) of the generators $\left(P_{i}, J_{j}, K_{k}\right)$, the $Q_{i}$ are found to be

$$
Q_{i}=i\left(\frac{\partial}{\partial p_{i}}+\frac{\alpha^{\prime} \beta-1}{2 \beta^{2}} p_{i}\right)
$$

In the Heisenberg representation the time evolution of the position operator is governed by

$$
\dot{Q}_{i}=i \beta^{\prime}\left(P_{0}\right)\left[P_{0}, Q_{i}\right]=\frac{P_{i}}{\beta},
$$

from which we conclude that

$$
p_{i}=\beta v_{i},
$$

where $v_{i}$ is the velocity of the particle. Note that we have formally written $\dot{Q}_{i}$ as the velocity of the particle and that the normalization $\dot{T}=1$ has been used. For massless particles we have $\beta^{2}=\vec{P}^{2}$, so that

$$
v=1 \text {, }
$$

where $v$ is the length of the velocity vector. Equation (67) yields, for a massive particle with mass $\mu$

$$
v_{\text {mas. part. }}<\left(1-\frac{\mu^{2}}{M^{2}}\right)^{\frac{1}{2}} \text {. }
$$

\section{Representations of the deformed Poincaré group}

The irreducible representations of the PA are associated with relativistic one-particle states [13]. Here we shall construct such representations for the dPA presented in section 2 and examine their relation with the corresponding representations of the ordinary PA.

Consider the quadratic Casimir invariant

$$
\beta^{2}\left(P_{0}\right)-P^{2}=\mu^{2}
$$

As in the ordinary PA there exist three classes of unitary representations: those with $\mu^{2}>$ $0, \mu^{2}=0$ and $\mu^{2}<0$. Let us denote the corresponding representations in each class as $[\mathrm{T}],[0]$ and $[\mathrm{S}]$, respectively.

For the $[\mathrm{T}]$-class we observe that we can choose a four-momentum $P_{\mu}$ to be

$$
P_{\mu} \propto( \pm 1,0,0,0)
$$

and there thus exist two subclasses in $[\mathrm{T}]$ denoted by $\left[\mathrm{T}_{ \pm}\right]$which correspond to the \pm choice of $P_{0}$. It is not difficult to see that the vector (70) is invariant under the little group $\mathrm{SO}(3)$ of this representation. Therefore these representations are classified according to Casimirs of $\mathrm{SO}(3)$, namely $S^{2}=s(s+1)$, where $s$ is the spin with values $s=0,1 / 2,1, \ldots$ As a result 
the one-particle states in the [T]-class are uniquely determined by the mass $\mu$, the spin $s$ and the $\operatorname{sign}(\beta)$.

In the [0]-class one can choose $P_{\mu}$ to be

$$
P_{\mu}=( \pm 1,0,0,1) \text {. }
$$

There thus exist in this case also two subclasses $\left[0_{ \pm}\right]$. The little group in this case is the two-dimensional Euclidean group $\mathrm{E}(2)$. Therefore the representations are here classified according to the Casimirs of $\mathrm{E}(2)$. These are the length of the two-dimensional vectors and the helicities of the helicity group $\mathrm{SO}(2)$, namely $0,1 / 2,1, \ldots$ Since the length of a two-vector can be zero or positive, the possible representations in the [0]-class can be $\left[0^{\circ}{ }_{ \pm}\right]$and $\left[0^{+}{ }_{ \pm}\right]$.

Finally, for the $[S]$-class we can choose

$$
P_{\mu} \propto(0,0,0,1)
$$

For this class the little group is the Lorentz group in three dimensions $\mathrm{SO}(1,2)$. The representations will be classified according to the Casimirs of $\mathrm{SO}(1,2)$.

$¿$ From the above discussion it is clear that there exists a correspondence between the representations of our $\mathrm{dPA}$ and the representations of the undeformed PA. This result would be immediately obtained if one noted that, by making the transformation $P_{0} \rightarrow \beta\left(P_{0}\right)$, the $\mathrm{dPA}$ is reduced to PA for the set of generators $\left(K_{i}, J_{i}, P_{i}, \beta\left(P_{0}\right)\right)$. However this transformation is not invertible, given the fact that $\beta$ is a multivalued function of $P_{0}$.

This last observation does not hold only in the present case. For instance in the case of $\mathrm{SU}_{q}(2)$ it is known that, in general, a redefinition of the $\mathrm{SU}(2)$ generators yields the $\mathrm{SU}_{q}(2)$ algebra and vice versa, when $q$ is not a root of unity [15]. Similarly in our case the redefinition of the generators $\left(K_{i}, J_{i}, P_{i}, P_{0}\right)$ of the dPA results in the ordinary PA. However this redefinition leaves the Lorentz subalgebra unaltered and thus the representation of the $\mathrm{dPA}$ are the same as in the usual PA. The only Casimir that is different in the two cases is the one defined in (32). Therefore the only difference in the one-particle states will appear in the masses labelling the representations.

\section{Discussion}

The hope that quantum theories with improved ultraviolet properties can be constructed has motivated a considerable research interest in deformations of space-time symmetries. However, in these studies one has to take into account that so far the laws of physics are required to be invariant under Lorentz transformations, while the notion of an elementary particle can be understood in the framework of the representation theory of the Poincare group. In this framework, consistent quantum field theories have been constructed; these describe successfully the non-gravitational interactions of elementary particles. In order to keep as much as possible of these features we were led to consider here minimal deformations of the space-time symmetries. Specifically in the present paper we were looking for deformations of the Poincare algebra that leave the Lorentz one invariant. As a result the representations of the constructed dPA are the same as in the ordinary case, modulo the mass. This fact is expected to play a catalytic role in the quantization of the field theories that one might attempt to construct. Moreover, the present construction introduces an upper limit in the 
energy. The deformed quadratic Casimir (32) and the upper bound in the energy provide also an upper bound in the momentum for free particles. On the other hand, in quantum field theories, loop diagrams involve exchanges of virtual particles that do not obey the on mass-shell condition (32). Then in these diagrams the fact that the energy is bounded relaxes by one unit the degree of their divergences. For instance, four-dimensional quantum field theories are expected to behave as three-dimensional ones from the renormalization point of view. This momentum space regularization can also be seen in the space-time as a regularization due to higher derivative terms obtained from the expansion of $\beta$ in powers of $P_{0}$. The latter leads to a non-local theory, which however is local in the momentum space. We shall discuss in detail the question of quantum field theories defined in the newly constructed framework in another publication.

We would like to thank L. Alvarez-Gaumé, H. Bacry, C. Bachas, A. Chakrabarti, E. Kiritsis, C. Kounnas and J. Madore for useful discussions. 


\section{References}

[1] U. Carow-Watamura, M. Schlieker, M. Scholl and S. Watamura, Int. J. Mod. Phys. A6 (1991)3081.

[2] P. Podleś and S.L. Woronowicz, Commun. Math. Phys. 130 (1990)381.

[3] W.B. Schmidke, J. Wess and B. Zumino, Z. Phys. C52 (1991)471;

O. Ogievetsky, W.B. Schmidke, J. Wess and B. Zumino, Lett. Math. Phys. 23 (1991)233; Commun. Math. Phys. 150 (1992)495;

M. Pillin and L. Weikl, "On representations of the q-deformed Lorentz and Poincaré algebra", preprint MPI-Ph/93-18, LMU-TPh 93-09.

[4] E. Celegnini, R. Giachetti, E. Sorace and M. Tarlini, J. Math. Phys. 31 (1990)2548; 32(1991)1155, 1159.

[5] J. Lukierski, H. Ruegg, A. Nowicki and V.N. Tolstoy, Phys. Lett. B 264 (1991)331;

J. Lukierski, A. Nowicki and H. Ruegg, Phys. Lett. B 293 (1992)344.

[6] M. Chaichian, J.A. de Azcarraza, P. Preŝnajder and F. Ródenas, Phys. Lett. B 281 (1992)41;

M. Chaichian and A.P. Demichev, Phys. Lett. B 304 (1993)220;

B. Aneva, "A realization of the quantum Lorentz group", preprint CERN-TH 7001/93.

[7] S. Sachse, "A deformed Poincaré algebra on a cubic lattice", preprint LMU-TPW 92-27; A. Schirrmacher, "Quantum groups, quantum spacetime and Dirac equation", preprint MPI-PTh/92-92.

[8] H. Bacry, J. Phys. A: Math. Gen. 26 (1993)5413; Phys. Lett. B 301 (1993)41; 306 (1993)44; 317 (1993)523.

[9] P. Maślanka, "Deformation map and Hermitean representations of the Poincaré algebra", Lodz preprint (April 1993).

[10] N.H. Christ, R. Friedberg and T.D. Lee, Nucl. Phys. B 202 (1982)89.

[11] V.G. Drinfeld, "Quantum groups", Proc. Int. Congress of Mathematicians (Berkeley, CA, 1986);

M. Jimbo, Lett. Math. Phys. 10 (1985)63; 11 (1986)247.

[12] J. Madore, Phys. Lett. B 263 (1991)245; Class. Quantum Grav. 9 (1992)69; Ann. Phys. 219 (1992)187;

A.A. Kehagias and G. Zoupanos, "Finiteness due to cellular structure of $R^{N}$ : I. Quantum mechanics", preprint MPI-Ph/93-59.

[13] E.P. Wigner, Ann. Math. 40 (1939)149.

[14] T.D. Newton and E.P. Wigner, Rev. Mod. Phys. 21 (1949)400;

M. Maggiore, "Quantum groups, gravity and the generalized uncertainty principle", preprint IFUP-TH 19/93. 
[15] P. Roche and D. Arnaudon, Lett. Math. Phys. 17 (1989)295;

G. Lusztig, Adv. Math. 70 (1988)237; Contemp. Math. 82 (1989)59;

T. Curtright and C. Zachos, Phys. Lett. B 243 (1990)237;

L. Mezincescu and R. Nepomechie, Phys. Lett. B 246 (1990)412;

L. Alvarez-Gaumé, C. Gomez and G. Sierra, Phys. Lett. B 220 (1989)142; Nucl. Phys. B 319 (1989)155;

A. Polychronakos, Mod. Phys. Lett. A 5 (1990)2325;

A. Chakrabarti, " $S O(5)_{q}$ and Contraction: Chevalley basis representations for q generic and root of unity", École Polytechnique preprint A.270.1193, Nov 1993. 